\title{
Lateral Inhibition Neural Networks for Classification of Simulated Radar Imagery
}

\author{
Charles M. Bachmann, Scott A. Musman, and Abraham Schultz \\ Code 5362, Airborne Radar Branch, Radar Division, Naval Research Laboratory \\ 4555 Overlook Ave., SW \\ Washington, D.C. 20375-5000 \\ January 8, 1992
}

\begin{abstract}
We investigate the use of neural networks for the classification of simulated inverse synthetic aperature radar (ISAR) imagery. Certain symmetries of the artificial imagery make the use of localized moments a convenient preprocessing tool for the inputs to a neural network. A database of simulated targets was obtained by warping dynamical models to representative angles and generating images with differing target motions. Ordinary backward propagation (bp) and some variants of bp which incorporate lateral inhibition obtain a generalization rate of up to $\sim 78 \%$ for novel data not used during training, a rate which is comparable to the level of classification accuracy that trained human observors obtained from the unprocessed simulated imagery.
\end{abstract}

\section{A Simulated ISAR Database}

Our database consisted of simulated inverse synthetic aperature radar (ISAR) images of ships. Real imagery is a Doppler vs. range profile produced by the ship's motion. Mathematically, the image shape depends on the cross-product of the line of sight vector with the instantaneous angular velocity vector of the ship. The resulting image exhibits the combined effects of the independent motions of the ship due to its roll, pitch, and yaw. Our artificial database contains target silhouettes which simulate the rudimentary shape of a ship in an image at different aspect angles and with varying degrees of roll, pitch, and yaw.

Although real imagery also has spectral characteristics, for the purposes of identifying the Perceptual Class of a ship, general shape information of a silhouette is usually sufficient. By "Perceptual Class" we mean the categories "Commercial/Auxilliary", "Combatant", "Landing Platform", "Submarine", and "Small Craft." For the purposes of this pilot study, we were interested in the determination of Perceptual Class. Therefore, we chose to simplify the problem by creating simulated images which all had binary image intensity levels.

We actually investigated a subset of the Perceptual Class problem: the ability of backward propagation and variants of bp incorporating lateral inhibition to distinguish the difference between commercial/auxilliary ships and combatants in the artificial database. For training, simulated images were presented to represent a variety of different target aspect angles and motion parameters. Each of these frames was presented once with the bow left and right. In figure 1, we show some examples of the kinds of image frames which we used.

The actual input to the network was the weighted rms image variation about the image center of mass in each range bin:

$$
\text { input }_{i}=\sqrt{\frac{\sum_{j}\left(j-E_{j}(i)\right)^{2} \rho_{i j}}{\sum_{i} \sum_{j}\left(j-E_{j}(i)\right)^{2} \rho_{i j}}}
$$

where $\rho_{i j}$ is the intensity of the image in range-doppler cell $i, j$, and $E_{i}(j)=\sum_{j} j \rho_{i j}$ is the image center-ofmass in range-bin i. Figure 2 shows a sample image frame and the corresponding range-bin moments used as input. Global 2-D invariant moments are of somewhat limited utility for recognition tasks such as this, which require more detailed shape information (Park and Sklansky, 1990). Therefore, we use local range-bin 

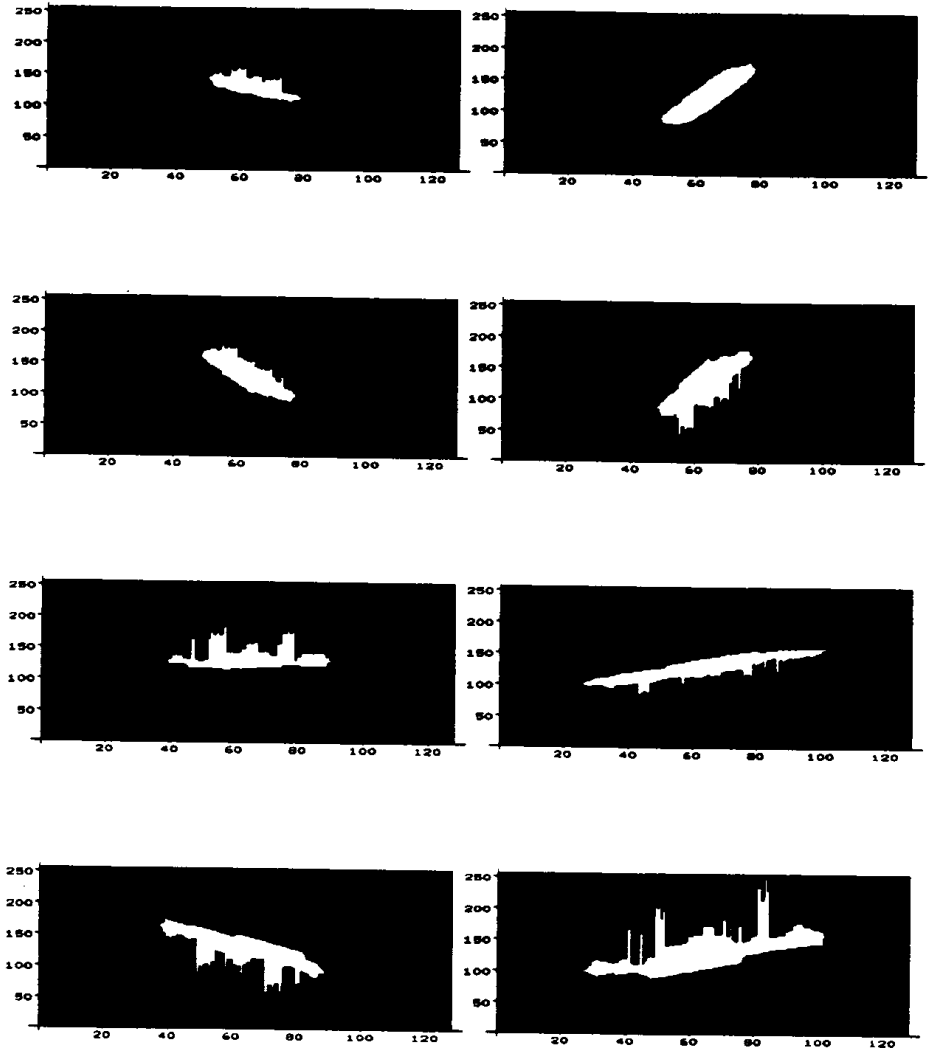

Figure 1: Examples of simulated binary ISAR images of a commercial/auxilliary ship. The same ship is displayed at a variety of different aspect angles, and with varying degrees of profile and plan component, which model the amount of roll, pitch and yaw present in the image.
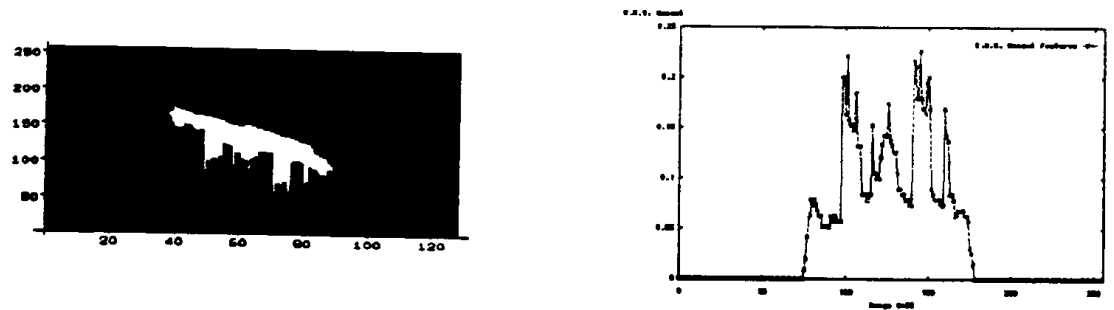

Figure 2: (Left) An example of a simulated ISAR silhouette. The horizontal axis is range, the vertical axis Doppler. (Right) The weighted rms variation about the image center of mass in each range bin is used as the input to the neural network. 
moments to give more details about the structural shape. We also make this choice because of symmetry: while features may change their scale and sign in the Doppler domain, they will remain constant in range.

Our database consisted of $\mathbf{4 8 9 6}$ simulated ISAR image frames drawn from 21 different ships. We divided the database into three subsets: one for training, a test set for monitoring generalization during the training procedure (cross-validation), and finally a second test set for evaluating generalization after training. Details of the composition of these data subsets are summarized in the following table.

\begin{tabular}{|c|cc|cc|}
\hline \multicolumn{5}{|c|}{ Table 1: Simulated ISAR Imagery Database } \\
\hline \hline Subset & Total Ships & $\begin{array}{c}\text { \# Commercial/Aux. } \\
\text { \# Combatants }\end{array}$ & \# of Frames & $\begin{array}{c}\text { \# Commercial/Aux. } \\
\text { \# Combatants }\end{array}$ \\
\hline Train & 9 & 5 & 2108 & 1020 \\
\hline & & 4 & 1496 & 1088 \\
Monitor & 6 & 3 & 1292 & 680 \\
\hline \multirow{2}{*}{ Novel } & 6 & 3 & 3 & 680 \\
\hline
\end{tabular}

\section{Lateral Inhibition Neural Networks}

Lateral inhibition has been studied in a variety of contexts, for example in unsupervised learning networks (Cooper and Scofield, 1988; Intrator, 1990; Seabach, 1990), in reinforcement learning (Sutton, personal communication) and dynamical models (Horn and Usher, 1990). Also, recent work (Giraud, Liu, bernard, and Axelrad, 1991) has looked at networks with excitatory-inhibitory pairs of neurons.

In the context of backward propagation (Rumelhart, Hinton, and Williams, 1986; Werbos, 1974), Sandon (1987) developed an ad hoc method for incorporating a linear competition between the error signals. At a given level in the network, the error signal for a particular neuron was modified by subtracting out an amount proportional to the sum of the error signals at the other nodes:

$$
\delta_{i}^{(n)} \rightarrow \delta_{i}^{(n)}-\frac{1}{N-1} \sum_{j=1}^{N}\left(\delta_{j}^{(n)}-\delta_{i}^{(n)}\right)
$$

Heuristically, it is appealing to allow competition between the error signals; however, such an approach does not guarantee that the network will implement a gradient descent minimization procedure. In contrast, we have taken the approach of imbedding the lateral inhibition in the energy functional and carrying out gradient descent on the modified energy functional. We accomplish this by introducing fixed lateral inhibition in the forward pass of the data through the network. With this approach, we have the advantage of being certain that we are actually carrying out a minimization procedure by gradient descent.

In our network, during the forward propagation of the pattern, the linear net input is computed for each cell, then the lateral inhibition of these inputs is computed, after which the nonlinear sigmoid is applied to the result to compute the firing rate of each cell. Therefore, the feedforward equations for pattern $s$ are:

$$
\begin{aligned}
& \tilde{x}_{i}^{s,(n)}=\sum_{j=1}^{N_{n-1}} w_{i j}^{(n-1)} \tilde{o}_{j}^{s,(n-1)}+\phi_{i}^{(n)} \\
& \tilde{o}_{k}^{s,(n)}=\sigma\left(\tilde{x}_{k}^{(n)}-\mu^{(n)} \sum_{j \neq k} \tilde{x}_{j}^{(n)}\right)
\end{aligned}
$$

where $\tilde{x}_{i}^{s,(n)}$ is the net feedforward input, $\tilde{o}_{k}^{s,(n)}$ is the cell activity, and $\sigma(x ; \lambda)=\frac{1}{1+e^{-\lambda=}}$ is the nonlinear sigmoid input-output function. Here, $\mu^{(n)} \equiv \frac{\alpha^{(n)}}{N_{n-1}}$ is the fixed parametric inhibition in layer $n$, and $N_{n}$ is the number of cells in that layer. Figure 3 compares the lateral inhibition architecture with that of ordinary backprop. With these modifications, the energy function to be minimized is defined in terms of the inhibited outputs of the final layer of the network. When we take the gradients of the modified energy functional with respect to the synaptic weights, we find that the ordinary backprop error signals, $\delta_{s i}^{(n)}$, must be replaced by inhibited error signals, $\tilde{\delta}_{s i}^{(n)}$, of the form:

last layer synapses : 


$$
\begin{aligned}
& \tilde{\delta}_{a i}^{(n)}=\gamma_{i}^{(n)}-\mu^{(n)} \sum_{k \neq i} \gamma_{k}^{(n)} \\
& \text { with } \\
& \gamma_{\alpha}^{(n)}=\left(\tau_{\alpha}^{*}-\tilde{o}_{\alpha}^{*,(n)}\right) \lambda_{\alpha}^{(n)} \tilde{o}_{\alpha}^{*,(n)}\left(1-\tilde{o}_{\alpha}^{s,(n)}\right)
\end{aligned}
$$

interior synaptic layers :

$$
\tilde{\delta}_{i}^{(n)}=\lambda_{i}^{(n)} \tilde{o}_{i}^{s,(n)}\left(1-\tilde{o}_{i}^{s,(n)}\right) \sum_{k} \tilde{\delta}_{k}^{(n+1)}\left(w_{k i}^{(n)}-\mu^{(n)} \sum_{j \neq i} w_{k j}^{(n)}\right)
$$

and the modification rule is written in terms of the inhibited error signal and inhibited neuronal input:

$$
\Delta_{s}\left(w_{k i}^{(n)}\right)=-\eta \frac{\partial \xi_{s(t)}}{\partial w_{k i}^{(n)}}=\eta \tilde{\delta}_{s k}^{(n+1)} \tilde{o}_{i}^{\alpha_{1}(n)}
$$

where $\xi_{s}=\sum_{k=1}^{N_{m}} \frac{1}{2}\left(\tilde{o}_{k}^{s,(m)}-\tau_{k}^{s}\right)^{2}$ is the LMS network error. Because the strength of the inhibitory connections is fixed, the inhibition appears parametrically in the expressions for the modified error signals. In fact, we can think of the inhibition equation as being equivalent to inserting a layer of non-modifiable connections $L_{i j}^{(n)}=\delta_{i j}^{(\text {Kronecker })}-\left(1-\delta_{i j}^{(\text {Kronecker })}\right) \mu^{(n)}$ between each layer of modifiable connections $w_{i j}^{(n)}\left(\delta_{i j}^{(K r o n e c k e r)}\right.$ is the Kronecker delta, or identity matrix). This fixed architectural constraint allows competition between the cells in a layer to represent particular features in the data.

Notice that the last layer error signals are equivalent to the ad hoc formula used by (Sandon, 1987); this layer is looking at the spatial difference in how close the output layer cells are to their respective targets. Our model differs from Sandon's in the interior layer: error signals for interior layers are propagated back across synaptic vectors which inhibit each other laterally. When $\mu^{(n)} \rightarrow \frac{1}{N_{n}}$, each cell is comparing itself to the average of all of the other cells in both the forward and backward passes. In contrast, as $\mu^{(n)} \rightarrow 0$, the algorithm reduces to the ordinary backprop network.
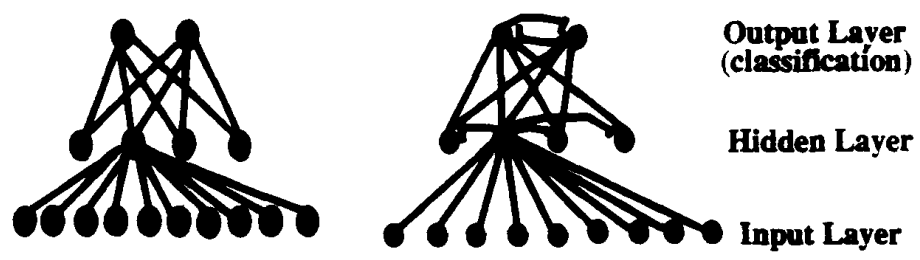

Figure 3: (Left) Ordinary backprop architecture. (Right) A backward propagation network with lateral inhibition. After the linear input in a layer is computed, the neurons laterally inbibit one another and then the nonlinear sigmoid is applied to obtain the actual cell response.

\section{Results}

\subsection{Benchmarks for Backprop and Lateral-Inhibition Variants}

We considered both three- and four-layer architectures (one and two layers of hidden units respectively). In the end, we settled on a three-layer architecture as the best configuration. Experiments with five hidden units revealed poor generalization to novel data. However, we obtained good generalization with seven or eight hidden units. More cells led to overfitting. Amplitudes of some sample first layer synaptic vectors are shown in figure 4.

We chose a small step-constant $\eta=0.1$ with moderate momentum $\kappa=0.6$ for the experiments. We trained the networks to distinguish between artificial images of combatants and those of commercial/auxiliaries as described above. Synapses were saved in a buffer whenever the generalization on the monitoring data set improved. The synapses in this buffer, representing the best level of generalization 

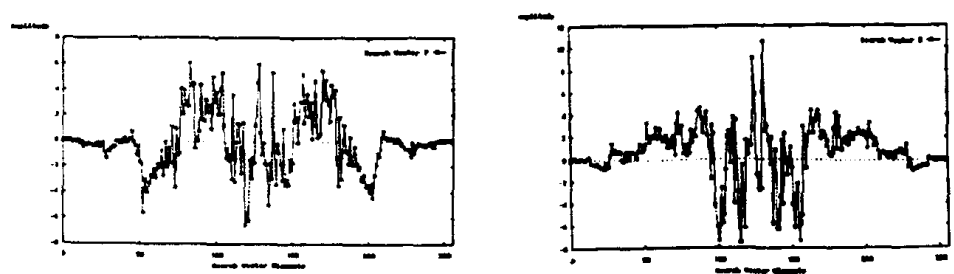

Figure 4: Examples of Amplitude plots for the elements of two first-layer synaptic vectors after training. Samples are from a 256-7-2 network configuration.

attained on the monitoring data set were eventually tested at the conclusion of each trial on the the third subset of the database as described above. The generalization results in the table are for this third independent database. This method of training was designed to compensate for the overtraining problem that is frequently encountered with backprop. By buffering the synapses only when generalization improves we lessen the chance that the network will overfit the data which often happens in the later phases of training. Such a training approach has been used by (Intrator, 1990) with BCM networks (Bienenstock, Cooper, and Munro, 1982) and in ordinary backprop (Bachmann, 1990).

\begin{tabular}{|c|c|c|c|c|c|}
\hline \multicolumn{6}{|c|}{$\begin{array}{l}\text { Table 2: Neural Network Gener } \\
\text { n of Range-Bin Moments of Sim }\end{array}$} \\
\hline$\alpha^{(n)}$ & trials & & & Mean & Beat \\
\hline (Inbibition) & & Combat. & Commerc. & Combined & Combined \\
\hline 0.0 & 20 & $84.0 \pm 5.8 \%$ & $54.5 \pm 5.8 \%$ & $69.2 \pm 5.2 \%$ & $77.6 \%$ \\
\hline 0.15 & 20 & $79.6 \pm 5.9 \%$ & $59.3 \pm 5.6 \%$ & $69.4 \pm 4.0 \%$ & $76.7 \%$ \\
\hline 0.25 & 20 & $82.5 \pm 7.4 \%$ & $55.0 \pm 8.0 \%$ & $68.8 \pm 3.4 \%$ & $76.4 \%$ \\
\hline 1.0 & 20 & $78.8 \pm 6.8 \%$ & $56.5 \pm 6.8 \%$ & $67.6 \pm 5.3 \%$ & $77.9 \%$ \\
\hline
\end{tabular}

\subsection{Understanding the Results}

There is not a significant difference in generalization over the range of inhibition levels which we explored with the neural networks. We feel that ordinary backprop is probably doing as well as can be expected given the limited number of ships in the database. Also, some of the viewing angles were extreme and therefore difficult to classify. Also, a number of frames had very little profile component in the image and therefore were not easily categorized. Furthermore, the initial representation using range-bin moments loses some of the shape information and could be improved. Obtaining generalization of up to $~ 78 \%$ ("Best Combined" column in Table 2), therefore, is a good result.

To better understand our results, we tested human observors trained in ISAR ship classification, asking them to classify the simulated binary images. A strict comparison is not really possible since our networks were trained with only a couple of thousand frames, whereas some of the people participating in the human tests had many years of experience in ISAR classification. Furthermore, the human observors looked at the raw images, not the range-bin moments. Nevertheless, their performance can give an indication of the difficulty of the problem. One hundred patterns were selected at random from our simulated database. The human observors were asked to classify the ships as either "commercial/auxilliary", "combatant", or "other (submarine, landing platform, or small craft)". Even though there were no submarines, landing platforms, or small craft present in the simulated images, this third perceptual category was provided as a means of estimating how often combatants and commercial / auxilliary ships would be confused with other perceptual classes.

\begin{tabular}{|c|c|c|c|c|c|c|}
\hline \multicolumn{7}{|c|}{$\begin{array}{l}\text { Table 3: Performance of Human Observors } \\
\text { Classification of Simulated ISAR Images (silhouettes) }\end{array}$} \\
\hline \multirow[t]{2}{*}{ Obervor } & \multicolumn{2}{|c|}{$\%$ Correct } & \multicolumn{2}{|c|}{ \% Misclassified as "other" } & \multicolumn{2}{|c|}{$\%$ Undecided } \\
\hline & Combat. & Commerc. & Combat. & Commerc. & Combat. & Commerc. \\
\hline$\overline{A L}$ & $70.9 \%$ & $42.2 \%$ & $18.2 \%$ & $37.8 \%$ & $0 \%$ & $0 \%$ \\
\hline WL & $83.6 \%$ & $31.1 \%$ & $12.7 \%$ & $20.0 \%$ & $1.8 \%$ & $2.2 \%$ \\
\hline $\mathrm{JM}$ & $89.1 \%$ & $48.9 \%$ & $5.5 \%$ & $13.3 \%$ & $5.5 \%$ & $15.6 \%$ \\
\hline LB & $92.7 \%$ & $60.0 \%$ & $5.5 \%$ & $15.6 \%$ & $0 \%$ & $0 \%$ \\
\hline DD & $89.1 \%$ & $66.7 \%$ & $7.3 \%$ & $6.7 \%$ & $0 \%$ & $0 \%$ \\
\hline MB & $98.2 \%$ & $80.0 \%$ & $1.8 \%$ & $2.2 \%$ & $0 \%$ & $0 \%$ \\
\hline Means: & $87.3 \pm 9.3 \%$ & $54.8 \pm 17.6 \%$ & $8.5 \pm 5.9 \%$ & $15.9 \pm 12.4 \%$ & $1.2 \pm 2.2 \%$ & $3.0 \pm 15.6 \%$ \\
\hline Both: & & & & $2 \%$ & & \\
\hline Best: & & & & $\%$ & & \\
\hline
\end{tabular}


Some of the variation can be accounted for by degree of experience. It is interesting, however, that the most experienced human observor, DD in the table, did not obtain the best score. Nevertheless, this experiment provides a baseline for the degree to which the perceptual class of the simulated image silhouettes can be identified.

The results which we have obtained for the neural networks for a range of randomly chosen initial starting conditions appear to have a similar mean classification accuracy, although we can not make a strict comparison because we have not allowed for an "other category" in the neural net experiments. An expanded database of "other" simulated targets will be useful in future work. We also plan to develop an on-line version of the simulation which will present randomly chosen views of the ships in the database to the neural network. Work on hybrid networks combining unsupervised and supervised learning algorithms is also planned.

\section{Acknowledgement}

We wish to acknowledge Dave Drake for assisting us in the creation of our artificial radar database and to thank the other members of the Airborne Radar Branch at NRL who participated in the human classification test. The theoretical results on lateral inhibition were completed at the Center for Neural Science of Brown University during the fall of 1990 by one of us (Bachmann) under the support of the National Science Foundation (grant \# EET 8719102), the Army Research Office (grant \# DAAL03-88-K-0116), and the Office of Naval Research (grant \# N00014-88-K-1316). The remainder of the work, including all of the simulations, was completed at the Airborne Radar Branch, Naval Research Laboratory, under the support of the Office of Naval Research (53-1501-0) and (53-1501-1).

\section{References}

[1] Bachmann, C. M., Learning and Generalization in Neural Networks, Ph.D. Dissertation, Brown University, Department of Physics, May 1990.

[2] Bienenstock, E. L., Cooper, L. N., Munro, P. W. Theory for the Development of Neuron Selectivity: Orientation Specificity and Binocular Interaction in Visual Cortex, The Journal of Neuroscience, Vol. 2, No. 1, pp. 32-48, January, 1982.

[3] Cooper, L. N., Scofield, C. L. Mean-Field Theory of a Neutal Network, Proceedings of the National Academy of Sciences USA, Vol. 85, pp. 1973-1977, March 1988, Neurobiology.

[4] Horn, D. and Usher, M., Excitatory-Inhibitory Networks with Dynamical Thresholds, International Journal of Neural Syustems, Vol. 1, No. 3 (1990), pp. 249-257.

[5] Intrator, N. I. Feature Extraction Using an Unsupervised Neural Network, in Proceedings of the 1990 Connectionist Models Summer School, Touretzky, D. S., Ellman, J. L, Sejnowski, T. J. (eds.), San Mateo, CA: Morgan Kaufmann.

[6] Giraud, B., Liu, L. C., Bernard, C., Axelrad, H., Optimal Approximation of Square Integrable Functions by a Flexible One-Hidden-Layer Neural Network of Excitatory and Inhibitory Neuron Pairs, Neural Networks, Vol. 4, pp. 803-815, 1991.

[7] Park, Y., Sklansky, J., Automated Design of Linear Tree Classifiers, Pattern Recognition, Vol. 23, No. 12, pp. 1393-1412, 1990.

[8] Rumelhart, D. E., Hinton, G. E., Williams, R. J. Learning Internal Representations by Error Propagation in Parallel Distributed Processing, Explorations in the Microstructure of Cognition, Vol. 1, Rumelhart, D. E., McClelland, J. L. (eds.), pp. 318-362, MIT Press: Cambridge, Mass., 1986.

[9] Sandon, P., Lecture on Extensions to Backward Propagation, G.T.E. Connectfest Conference, Waltham, Mass., Sept. 25, 1987.

[10] Seebach, B. S., Evidence for the Development of Phonetic Property Detectors in a Modified BCM Neural Network without Innate Knowledge of Linguistic Structure, Ph. D. Dissertation, Brown University, Program in Neural Science, 1990.

[11] Werbos, P. J., Beyond Regression: New Tools for Prediction and Analysis in Behavioral Sciences. Ph.D. Thesis, Harvard University, 1974. 